\title{
Cardiac amyloidosis with gastrointestinal involvement: a case report.
}

\author{
Alina Tantau1, Ioan Avram², Angela Cozma1, Dorel Sampelean'1 \\ 14th Medical Department, University of Medicine and Pharmacy "Iuliu Hatieganu", ${ }^{2}$ Railway Hospital Cluj Napoca, \\ Romania
}

\begin{abstract}
Familial amyloidosis is a rare type of amyloidosis, difficult to diagnose. We present the case of a woman with chronic heart failure. Low ejection fraction and concentric left ventricle hypertrophy with granular sparkling were seen by echocardiography and cardiac magnetic resonance imaging. Based on myocardial biopsy and genetic tests, the diagnosis of transthyretin familial amyloidosis, secondary to the Glu54Gln gene mutation, was made. The presentation contains the diagnostic algorithm used in the case of our patient, including clinical, biochemical, imaging, histological and genetic examinations, for the purpose of a complete diagnosis.
\end{abstract}

Keywords: amyloidosis, restrictive cardiomyopathy, transthyretin

\section{Introduction}

Hereditary transthyretin amyloidosis is a rare type of amyloidosis, secondary to mutations in the genes involved in transthyretin synthesis $[1,2]$. The clinical presentation varies greatly, including polyneuropathy and cardiomyopathy [1]. The diagnostic of cardiac amyloidosis should be suspected when a hypertrophic or restrictive cardiomyopathy is detected $[3,4]$. A histologic specimen for confirmation of amyloidal deposits is mandatory for an accurate diagnostic [2].

We presented a case of young women with suspected cardiac amyloidosis on echocardiography and cardiac magnetic resonance imaging (MRI). Autonomic neuropathy of the digestive tract was also suspected, but the gastric and rectum biopsies were negative for amyloid. Finally, amyloid infiltration of myocardium on cardiac biopsy was demonstrated and a molecular genetic profile was made.

Received 17.07.2014 Accepted 09.09.2014

Med Ultrason

2015, Vol. 17, No 1, 123-125

Corresponding author: Tantau Alina,

18, Republicii street, Cluj Napoca, Romania

Phone: +40751110150

Fax: +40264598278

Email: alitantau@gmail.com

\section{Case report}

A 52-year-old female patient presented for a cardiological examination complaining of a marked decrease of exercise tolerance, effort dyspnea, and palpitations, symptoms with progressive onset. The patient was in general good state, with a blood pressure $110 / 70 \mathrm{mmHg}$, a regular ventricular rate of $80 \mathrm{bpm}$ and peripheral cyanosis. The electrocardiogram evidenced a sinus rhythm, negative T waves in DII, DIII and avF leads, microvoltage, and a P-R interval of $0.2 \mathrm{~mm}$. Cardiac ultrasound (Healthcare GE VIVID S6 BT08 machine) detected concentric cardiac hypertrophy, hyperechogenity with a bright and granite-like appearance, secondary to amyloid deposits of left ventricular wall and interventricular septum, thickening of the cardiac wall, with infiltrated mitral valve and tricuspid valve leaflets, diastolic restrictive dysfunction, atrioventricular regurgitation, and dilated left and right atrium without changes of ventricular chambers (fig 1, fig 2). The aspect was specific for restrictive dysfunction. Also, thickened and infiltrated interatrial septum and pericardial fluid collection without hemodynamic significance were found. The interpretation was of restrictive cardiomyopathy (RCM), the suspicion of amyloidosis being raised. The cardiac MRI confirmed the RCM with diffuse infiltrative changes in the left ventricular myocardium and the interventricu- 


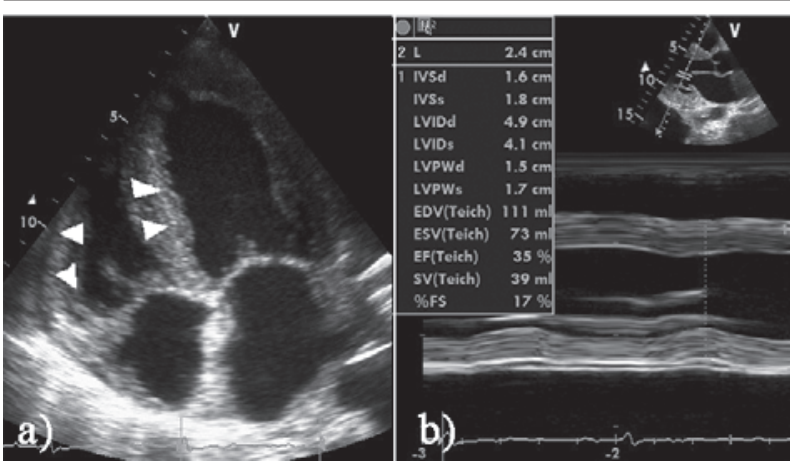

Fig 1. a) Four chambers section: hyperechoic with a bright and granite-like appearance, secondary to amyloid deposits of the left ventricular wall and interventricular septum can be seen; b) M-mode examination: thickening of the cardiac wall.

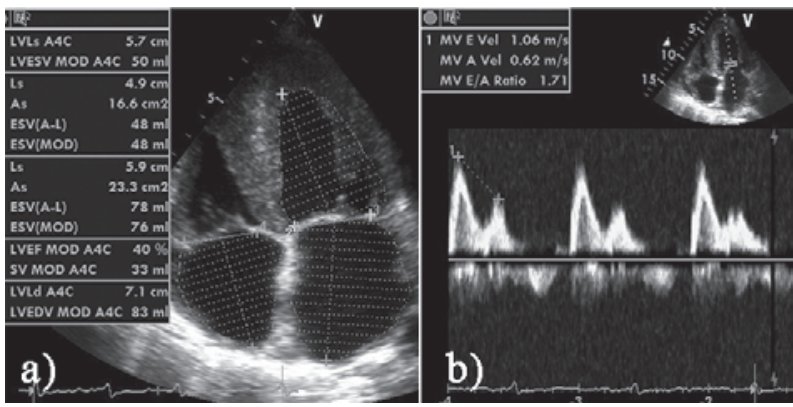

Fig 2. a) Pulsate wave Doppler: diastolic restrictive type dysfunction at the level of mitral valve is demonstrated; b) cardiac measurement of the cardiac chambers. Normal dimensions of the ventricles but with increasing of atrial chambers dimensions, typical for restrictive dysfunction.

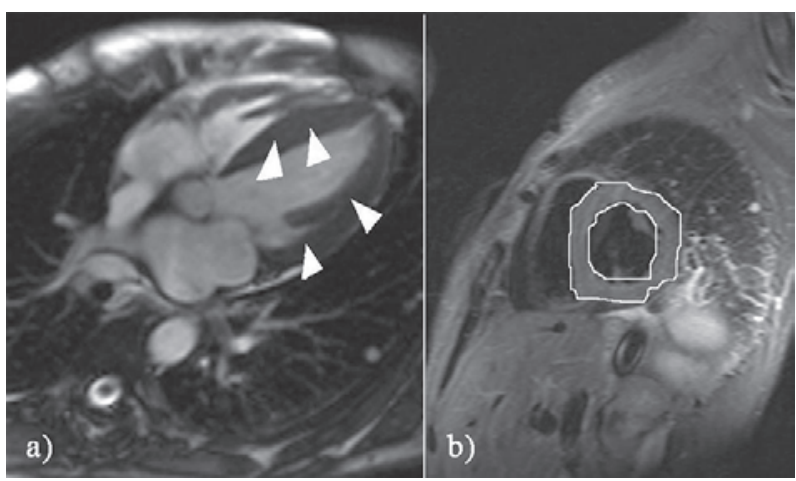

Fig 3. Cardiac MRI: a) Infiltrative changes in the left ventricular myocardium and the interventricular septum; b) Thickening of the ventricular wall secondary of amyloid deposit.

lar septum, as well as the presence of infiltrates in the other cardiac walls (fig 3), with a predominantly subendocardial location, the resulting appearance suggesting cardiac amyloidosis. Conservative management of the case with diuretics and beta blockers (bisoprololum) was started and the patient was discharged, no other test being performing at that time.
Six months later, the patient was admitted with nausea, vomiting, pyrosis, regurgitation, diarrhea (6 watery stools/day), paresthesia of the lower and upper limbs, fatigue, weight loss, polyarthralgia (hands, feet, and spine) with morning stiffness, symptoms with a relatively sudden onset of several days. The clinical examination detected dehydrated skin and mucosae, peripheral cyanosis, mild hepatomegaly, first degree splenomegaly and diminished rotulian and Achilles reflexes. Mild thrombocytopenia, discrete normocytic anemia, and mild hypergammaglobulinemia were found in laboratory tests. Abdominal ultrasound detected mild stasis hepatomegaly, mild splenomegaly, and normal kidney. Colonoscopy and gastroduodenoscopy were negative and rectal/ gastroduodenal biopsy detected no amyloid deposits in the submucosa. The electromyogram diagnosed chronic axonal sensory-motor polyneuropathy, probably related to amyloidosis.

The diagnosis of systemic amyloidosis was finally established after abdominal fat and myocardial biopsies in which the amyloid deposits were confirmed. The genetic tests evidenced the Glu54Gln gene mutation. The final diagnosis was transthyretin familial amyloidosis p(ATTR) secondary to the Glu54Gln gene mutation, restrictive cardiomyopathy, peripheral and autonomic polyneuropathy of the digestive tract. The patient was proposed for both heart and liver transplantation.

\section{Discussions}

ATTR is a rare type of amyloidosis, being most frequently caused by a protein called transthyretin (TTR) [1]. ATTR may present with highly variable symptoms, including polyneuropathy and cardiomyopathy [3]. Amyloid infiltrates any or all of the cardiovascular structures, including the conduction system, the atrial and ventricular myocardium, valvular tissue, and the coronary and large arteries, leading to arrhythmia, global heart failure and death [3].

We present the case of a patient with ATTR and gastrointestinal (GI) involvement due to autonomic neuropathy. In this case, cardiac ultrasound and MRI provided important information by evidencing two aspects suggestive of amyloidosis, i.e. restrictive cardiomyopathy and wall hyperechogenicity, with a bright and granite-like appearance, secondary to amyloid deposits [2-4]. Myocardial infiltration progressively increases the thickness of the left and right ventricular walls and the interventricular septum [3-6]. Infiltrated mitral and tricuspid valve leaflets, bilateral atrial dilation and thickened atrial septum are frequent in amyloidosis but no specific [5,6]. Cardiac amyloidosis is generally considered to be a cardiomyo- 
pathy; a restrictive filling pattern is patognomonic of the disease process but is seen only in advanced stages $[5,6]$. The majority of patients present with symptoms of congestive heart failure. Half of the patients are diagnosed with pericardial effusion [3].

GI complications are common in ATTR and the autonomic neuropathy has been considered to explain the symptoms. Diarrhea, nausea and vomiting are often present [7]. The sensitivity of endoscopic biopsy of the gastrointestinal mucosa is approximately $85 \%$ [8]. Our patient had GI symptoms and autonomic neuropathy of the digestive tract was suspected but repeatedly biopsies from stomach and rectum were negative.

Theoretically, typical echocardiographic appearances would be enough to diagnose cardiac amyloidosis when it is impossible for the patient to undergo a cardiac biopsy, if an additional histological specimen from another tissue such as rectal mucosa or abdominal fat confirms amyloidosis [6]. In our case, abdominal fat biopsy was positive for amyloid deposits. Even so, the experts in the field had recommended a histological diagnosis from the endomyocardial biopsy, which in fact is extremely sensitive and specific, the biopsy material having the same aspect as that described for the amyloid [1,2]. Furthermore, molecular genetic testing was essential in the diagnostic strategy [6].

From a therapeutic point of view, the main objective is to prevent the tissue deposition of the amyloid and to favor the resorption of deposits [2]. Currently, the first line treatment in the case of patients with ATTR is liver transplantation, which prevents the production of mutated transthyretin [9]. Gene therapy also promises strategies in these patients and various clinical studies have been launched [10].

In conclusion, ATTR is a rare type of amyloidosis. Imagistic findings consist of cardiac amyloidosis and are recognizable by the cardiac ultrasonographer and radi- ologist. In our case a complete diagnosis was made by histological and genetic tests.

Acknowledgement: This paper was published under the frame of the European Social Fund, Human Resources Development Operational Programme 2007-2013, POSDRU/159/1.5/S/138776.

\section{References}

1. Morie A, Gertz SVR. Amyloidosis: Diagnosis and Treatment. Londra: Humana Press, 2010.

2. Maria M, Picken MD, Ahmet D, Guillermo AH. Amyloid and Related Disorders. New York: Humana Press, 2012.

3. Falk RH, Dubrey SW. Amyloid heart disease. Prog Cardiovasc Dis 2010; 52: 347-361.

4. Elliott P, Andersson B, Arbustini E, et al. Classification of the cardiomyopathies: a position statement from the European Society of Cardiology Working Group on Myocardial and Pericardial Diseases. Eur Heart J 2008; 29: 270-276.

5. Banypersad SM, Moon JC, Whelan C, Hawkins PN, Wechalekar AD. Update in cardiac amyloidosis: a review. J Am Heart Assoc 2012; 1: e000364.

6. Falk RH. Diagnosis and management of the cardiac amyloidosis. Circulation 2005; 112: 2047-2060.

7. Suhr OB, Svendsen IH, Andersson R, Danielsson A, Holmgren G, Ranlov PJ. Hereditary transthyretin amyloidosis from a Scandinavian perspective. J Intern Med 2003; 254: 225-235.

8. Guy CD, Jones CK. Abdominal fat pad aspiration biopsy for tissue confirmation of systemic amyloidosis: Specificity, positive predictive value, and diagnostic pitfalls. Diagn Cytopathol 2001; 24: 181-185.

9. Sekijima Y. Recent progress in the understanding and treatment of transthyretin amyloidosis. J Clin Pharm Ther 2014; 39: 225-233

10. Penchala SC, Connelly S, Wang Y, et al. AG10 inhibits amyloidogenesis and cellular toxicity of the familial amyloid cardiomyopathy-associated V122I transthyretin. Proc Natl Acad Sci U S A 2013; 110: 9992-9997. 